\title{
Schlechte Zeit für Mythen Zu Brechts Lyrik
} des Exils

Temps difficiles pour les mythes. À propos des poèmes d'exil de Bertolt Brecht Bad Time for Myths. On Brecht's Lyrics of Exile

\section{Lutz Hagestedt}

\section{(2) OpenEdition}

Journals

Édition électronique

URL : http://journals.openedition.org/ceg/4531

DOI : 10.4000/ceg.4531

ISSN : 2605-8359

\section{Éditeur}

Presses Universitaires de Provence

\section{Édition imprimée}

Date de publication : 2 mai 2019

Pagination : 121-134

ISBN : 979-10-320-0214-8

ISSN : 0751-4239

\section{Référence électronique}

Lutz Hagestedt, "Schlechte Zeit für Mythen Zu Brechts Lyrik des Exils", Cahiers d'Études Germaniques

[Online], 76 | 2019, Online erschienen am: 02 November 2019, abgerufen am 24 Januar 2021. URL: http://journals.openedition.org/ceg/4531 ; DOI: https://doi.org/10.4000/ceg.4531 


\section{Schlechte Zeit für Mythen $\mathrm{Zu}$ Brechts Lyrik des Exils}

Lutz HAGESTEDT

Universität Rostock

Fragt man sich, wer in Bezug auf die Mythos-Rezeption Vorbild für Brecht gewesen sein könnte, so fallen einem die ,Klassiker ein. Darunter verstand Brecht die ,philosophischen Klassiker Marx und Engels sowie deren Epigonen Lenin, und sie treten als solche in seiner Lyrik auch auf. Marx und Engels werteten Religion als ,Opium des Volkes und standen den heidnischen und christlichen Mythen entsprechend skeptisch gegenüber. Gern wird der folgende Passus aus der Deutschen Ideologie zitiert, die vor allem Karl Marx 1845/46 verfasste:

\footnotetext{
Die ganze Mythologie der selbständigen Begriffe, mit dem Wolkensammler Zeus, dem Selbstbewußtsein, an der Spitze, paradiert hier wieder mit „dem Schellenspiel von Redensarten einer ganzen Janitscharenmusik gangbarer Kategorien“ [...]. Zuerst natürlich die Mythe von der Weltschöpfung, nämlich von der sauren „Arbeit“ des Kritikers, die das „einzig Schöpferische und Produzierende, ein immerwährendes Kämpfen und Siegen, ein fortdauerndes Vernichten und Schaffen“, ein „Arbeiten“ und „Gearbeitet-Haben“ ist. ${ }^{1}$
}

Hier fallen Begriffe, die für Brecht von zentraler Relevanz gewesen sind. Denn wie vielleicht kein zweiter Lyriker des 20. Jahrhunderts hat er Aspekte der Arbeit und des Kampfes, der Schöpfung und der Zerstörung, der Verhältnisse und ihrer Kritik ins Zentrum seines Werkes gestellt. Einige seiner Gedichte thematisieren diese Gegensätze in einer solchen Schlichtheit, dass man sie beinahe für Kindergedichte halten möchte. Zum Beispiel „Das Lied vom Sankt Nimmerleinstag“ oder „Das Lied von der Wehrlosigkeit der Götter und Guten“ - beide 1940 entstanden. Das eine spielt auf die christliche Vorstellung vom Jüngsten Gericht an, das andere stellt die Theodizee-Frage, und beide wurden in sein Stück Der gute Mensch von Sezuan (1943) eingebunden. ${ }^{2}$ Beide Gedichte demonstrieren im Grunde, dass die christlichen Heilsversprechen unerfüllt

1. Karl Marx, Friedrich Engels, „Sankt Bruno contra die Verfasser der „Heiligen Familie““, in dies., Die deutsche Ideologie. Kritik der neuesten deutschen Philosophie in ihren Repräsentanten, Feuerbach, B. Bauer und Stirner, und des deutschen Sozialismus in seinen verschiedenen Propheten, Berlin, Dietz, 1953, S. 96-104, hier S. 98. Wie schon in seiner ersten Schrift, Die heilige Familie (1845), für die er gemeinsam mit Engels zeichnete (wobei Engels ,fast nichts“ dazu beitrug), polemisiert Marx hier gegen seinen Lehrer, den Junghegelianer Bruno Bauer.

2. Bertolt Brecht, „Das Lied von der Wehrlosigkeit der Götter und Guten“, in ders., Gedichte 5. Gedichte und Gedichtfragmente, 1940-1956 (= Bd. 15 der Großen kommentierten Berliner und Frankfurter Ausgabe; im folgenden kurz GBA 15), hrsg. von Werner Hecht, Jan Knopf, 
bleiben: die Lehren vom guten Gott, vom Jüngsten Gericht, von der Vergebung der Sünden, von der Auferstehung der Toten sowie vom Ewigen Leben bleiben ,Mythen“, und erst „Am Sankt Nimmerleinstag wird die Erde zum Paradies.“ ${ }^{3}$ Also niemals.

Mythenkritik und Mythenrevisionen sind konstitutive Bestandteile des Brecht'schen Euvres. An Religionen hat er nicht geglaubt, aber auf das biblische (und heidnische) Mythenreservoir hat er oft zurückgegriffen, um ethische und soziale Aspekte der Daseinsbewältigung zu illustrieren. Schon in seinem ,Urbaal (1918) setzte er auf die Wirkungsmacht alter Mythen und schrieb sich - im Rückbezug auf François Villon - in eine Traditionslinie der Moderne ein (Hanns Johst, Johannes R. Becher, Georg Heym und andere wären hier zu nennen). Im Spätwerk nutzte Brecht das Instrument der Mythisierung zur Überhöhung des Politischen - er schuf sich im Kommunismus eine weltanschauliche Ersatzreligion und verlor dabei seinen neusachlich-nüchternen Blick für die gegenüber dem Ideal defiziente Realität.

Da die Prosa und die Dramatik bereits des Öfteren in den Fokus literaturwissenschaftlicher Betrachtung gerückt sind - vor allem unter dem Aspekt der „Mythenkorrektur“ -, beschränke ich mich weitgehend auf die Lyrik, die bei Brecht vielfach ebenfalls im erzählenden und im dramatischen Modus gehalten ist. ${ }^{4}$ Und wo Brecht seine Gedichte argumentativ einsetzt, kommt das ,Prosaische“ (respective ,Dialogische') seiner Lyrik der spezifischen Form seiner Mythenrezeption entgegen. Eine nach wie vor vorzügliche Einführung und Einstimmung in den Mythologiker Brecht gibt uns Franco Buono, der in einem Aufsatz Brechts Prosa-Etüden „Berichtigungen alter Mythen“ (1933) untersuchte, und der in einem zweiten Beitrag Brechts Mythosrezeption mit der Brecht'schen Marxismusrezeption korrelierte. ${ }^{5}$ Franco Buono hat schon früh auf die kongeniale „Mythologie“ der Brecht'schen „Berichtigungen“ verwiesen und sie den Mythenkontrafakturen Kafkas an die Seite gestellt. Er sah in ihnen

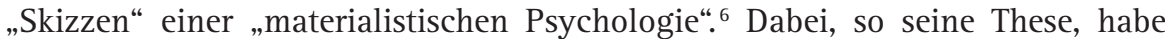
nicht erst das dänische Exil eine „Begegnung“ Brechts „mit der Antike und der klassischen Tradition“ gezeitigt. Nein, Brecht sei bereits 1923/24 mit antiken Mythen schöpferisch befasst gewesen:

aber sicher war es eine Zeit, in der er lebhaft das Bedürfnis spürte, auf Geschichte und Traditionen zurückzublicken, ihnen die Krusten abzukratzen, die sie verdächtig und unbrauchbar machten, und statt des antiken einen neuen Begriff von Geschichte und Klassizität zu schaffen. Um den Zeitpunkt noch genauer zu bestimmen, müssen wir

Werner Mittenzwei und Klaus-Detlef Müller, Berlin/ Weimar/ Frankfurt a. M., Aufbau und Suhrkamp, 1993, S. 8 f. Vgl. dazu den Brecht-Kommentar, 319 f.

3. Ebd.

4. Vgl. etwa Otto Mann, B. B. - Maß oder Mythos? Ein kritischer Beitrag über die Schaustücke Bertolt Brechts, mit einem Geleitwort des Verlegers, Heidelberg, Rothe, 1958.

5. Franco Buono, „Odysseus, Kandaules, Ödipus und Brecht“, in ders., Zur Prosa Brechts, Aufsätze. Aus dem Italienischen von Maria Böhmer-Volo, Frankfurt a. M., Suhrkamp, 1973 (suhrkamp taschenbuch, 88), S. 61-91. Sowie ders., „Bemerkungen über Marxismus und Geschichte bei Bertolt Brecht“, ebd. S. 92-120.

6. Franco Buono, „Odysseus...“, S. 62. 
uns ins Gedächtnis rufen, daß ebenfalls aus dem dänischen Exil Die Horatier und die Kuratier (1933-34) stammen, die, wenn auch in der strengen Form des Lehrstücks, ein anderes berühmtes Beispiel darstellen, bei dem Geschichte und klassische Mythologie zusammenfließen. $^{7}$

Als überzeugenden Aufhänger seiner Betrachtungen wählte sich Buono die siebte der „Geschichtsphilosophischen Thesen“ Walter Benjamins, die von „Kulturgütern“ im Zeichen der großen Raubzüge handelt. Man könne, so Benjamin, ein als „Beute“ im Triumphzug mitgeführtes Kulturgut „nicht ohne Grauen“ betrachten, da seine Aneignung immer auch das Signum der Barbarei in sich trage: „Und wie es selbst nicht frei ist von Barbarei, so ist es auch der Prozeß der Überlieferung nicht, in der es von dem einen an den anderen gefallen ist.“8

Spricht Benjamin von Barbarisierung, die zum „kulturellen Erbe“ und seiner Aneignung gehöre, so muss man, mit Blick auf Brecht, auch an dessen Programm der ,Vulgarisierung“ und ,Proletarisierung“ rühren. Denn es ist ein unübersehbarer Zug seiner Mythenrezeption, den Mythos ,tendenziös‘ zu deuten und ostentativ gegen den Strich zu bürsten, so zwar, dass der Zivilisationsbruch sichtbar wird, den die Moderne im Umgang mit der Tradition in Kauf genommen, wenn nicht betrieben hat. Brechts Lyrik setzt diesen Zivilisationsbruch vielfach schon voraus - im Rückblick als sich ereignet habenden, in visionärer Schau als bevorstehenden. Seine Gedichte führen dabei vor, dass die Kriegswirklichkeit der Moderne (es ist von „Tanks und Kanonen / Schlachtschiffe[n] und Bombenflugzeuge[n] und Mine[n]“ die Rede) mit der christlichen Mythologie nicht in Einklang zu bringen sei. ${ }^{9}$ Eine Erfahrung, die vermutlich alle Dichter gemacht haben dürften, die eine Heilsbotschaft an der Wirklichkeit gemessen haben. Als Beispiel mag Percy Bysshe Shelley fungieren, dessen Gedicht „The Hell“ (1819) Brecht im Kontext seiner Hollywood-Elegien thematisierte:

Nachdenkend, wie ich höre, über die Hölle

Fand mein Bruder Shelley, sie sei ein Ort

Gleichend ungefähr der Stadt London. Ich

Der ich nicht in London lebe, sondern in Los Angeles

Finde, nachdenkend über die Hölle, sie muß

Noch mehr Los Angeles gleichen.

Auch in der Hölle

Gibt es, ich zweifle nicht, diese üppigen Gärten

Mit den Blumen, so groß wie Bäume, freilich verwelkend

Ohne Aufschub, wenn nicht gewässert mit sehr teurem Wasser.

[...] Und endlose Züge von Autos

Leichter als ihr eigener Schatten, schneller als

Törichte Gedanken, schimmernde Fahrzeuge, in denen

Rosige Leute, von nirgendher kommend, nirgendhin fahren.

Und Häuser, für Glückliche gebaut, daher leerstehend

7. Buono, „Odysseus...“, S. 64. Der Verfasser weist uns auch auf die „denkwürdige Rede Mortimers auf den trojanischen Krieg“ hin, die 1923 verfasst worden war.

8. Walter Benjamin, Über den Begriff der Geschichte (1940). Zit. nach Buono, „Odysseus...“, S. 65. Benjamin spricht hier vom „Dokument der Kultur“, das „zugleich ein solches der Barbarei“ sei.

9. Brecht, Das Lied von der Wehrlosigkeit der Götter und Guten. In ders., Gedichte 5, GBA 15, S. 8 f. Hier: S. 8. 
Auch wenn bewohnt.

Auch die Häuser in der Hölle sind nicht alle häßlich.

Aber die Sorge, auf die Straße geworfen zu werden Verzehrt die Bewohner der Villen nicht weniger als Die Bewohner der Baracken. ${ }^{10}$

Die Stadt der Engel gewährt den Hütten keinen Frieden, aber sie stimuliert auch keine Änderungserwartung, die einen Krieg den Palästen entfesselte. Diese Hölle auf Erden ist mit ihrer Kontingenz der Zustände und Verläufe nicht steuerbar - wie von unsichtbarer Hand geführt, aber sinnlos und undurchschaubar schlängeln sich die Wagenkolonnen durchs Tal. Die besondere Perspektive des Exilierten ist immer auch selektiv: Apokalyptisch leerstehende Häuser, in denen kein Glück zuhause ist, sind bewohnt und wirken doch unbehaust. Die Hölle, das sind die anderen - im paradoxalen System des Kapitalismus manifestieren sich die „Wirtschaftssorgen eines Arbeiters“ primär als „Sorgen eines Konsumenten“: Wird er „sein Haus halten“ können, „wenn die Hypothekenzinsen steigen?“ "11 Die existenziellen Fragen erweisen sich im Zeichen des Wohlstands als nicht weniger dringlich.

Natürlich hat Brecht an die christliche Mythe der Verdammnis nicht geglaubt - weder die paradiesischen Gärten noch die Phantasmagorien der Höllenqualen wurden von ihm ernstlich erwogen. „Welch ein Betrug!“ ruft er emphatisch aus. ${ }^{12}$ Glauben heißt für Brecht, „der Menschen Wahn ins Schwarze schrauben!" ${ }^{13}$ Daher erklärt er die Welt der Bibel zum poetischen Spielmaterial, zur Verfügungsmasse kultureller Gestaltung, die nicht der Glaubenspraxis bedürfe, sondern dem Glaubenszweifel (und damit der Gegenprobe) Rechnung trage. Denn oft genug hält die Mythe der Wirklichkeit nicht stand, wie sein Gedicht „Die Schrift sagt, sie steht still“ (1938) und noch sein Galilei (1939) belegen. ${ }^{14}$ In erster Linie repräsentiert das Buch der Bücher für Brecht einen kanonischen Erzählfundus literarischer Traditionsstiftung, denn es hat von jeher die Dichter zu eigenen Heils- und Unheilsgesängen angeregt, etwa einen Dante zu seiner „Hölle der Abgeschiedenen“, einen Milton zu Paradise Lost oder einen Shelley zu seinem Höllengedicht. ${ }^{15}$ In diese Tradition schreibt sich der Augsburger mit seinem „Salomon Song“ ein:

Ihr saht den weisen Salomon

10. Brecht, Nachdenkend, wie ich höre, über die Hölle, in ders., Gedichte 5, GBA 15, S. 46. Kommentar, S. $340 \mathrm{f}$.

11. Vgl. Niklas Luhmann, Die Wirtschaft der Gesellschaft. Frankfurt a. M., Suhrkamp, 1988, S. 164.

12. Brecht, Kritik an Michelangelos „Weltschöpfung“, in ders., Gedichte 4, Gedichte und Gedichtfragmente, 1928-1939 (= Bd. 14 der Großen kommentierten Berliner und Frankfurter Ausgabe; im folgenden kurz GBA 14), hrsg. von Werner Hecht, Jan Knopf, Werner Mittenzwei und Klaus-Detlef Müller, Berlin/ Weimar/ Frankfurt a. M., Aufbau und Suhrkamp, 1993, S. 440 f. Hier: S. 421. Kommentar, S. 670.

13. So in Brechts Gedicht „Kritik an Michelangelos ,Weltschöpfung““, in ders., Gedichte 4, GBA 14, S. 420 f. Hier: S. 420.

14. Vgl. Brecht, „Die Schrift sagt, sie steht still“ (um 1938), in ders., Gedichte 4, GBA 14, S. 420.

15. Vgl. Brecht, „Der Augsburger geht mit Dante durch die Hölle der Abgeschiedenen“ (um 1938), in ders., Gedichte 4, GBA 14, S. 417. 
Ihr wißt, was aus ihm wurd.

Dem Mann war alles sonnenklar

Er verfluchte die Stunde seiner Geburt

Und sah, daß alles eitel war. [...]

Ihr saht den kühnen Cäsar dann

Ihr wißt, was aus ihm wurd!

Der saß wie'n Gott auf dem Altar

Und wurde ermordet, wie ihr erfuhrt

Und zwar, als er am größten war.

Wie schrie der laut: auch du, mein Sohn! [...]

Ihr kennt den redlichen Sokrates

Der stets die Wahrheit sprach:

Ach nein, sie wußten ihm keinen Dank

Vielmehr stellten die Obern böse ihm nach

Und reichten ihm den Schierlingstrank. [...]

Hier seht ihr ordentliche Leut

Haltend die zehn Gebot

Es hat uns bisher nichts genützt

Ihr, die am warmen Ofen sitzt

Helft lindern unsre große Not! [...] ${ }^{16}$

Als „Ballade von den Prominenten“ ist Brechts Gedicht von Kurt Weill vertont worden und in die Dreigroschenoper eingegangen. ${ }^{17}$ Der "Song“ stammt also schon aus der ,Systemzeit", wurde in hoher Auflage (wohl um die 10000 Exemplare) im Oktober 1928 gedruckt und im Exiljahr 1937 für die Gesammelten Werke (bei Malik) neu gefasst. ${ }^{18}$ Das Gedicht korreliert ein Ensemble mythischer und historischer Personen (Salomon, Cäsar, Sokrates), die an ihren Tugenden (Weisheit, Kühnheit, Wahrheitsliebe) zerbrechen, mit einem Kollektiv der Gottesfürchtigen (als „ordentliche Leut“ apostrophiert), die nach dem Dekalog leben und an dessen Wirklichkeitsfremdheit scheitern. Der Mensch tritt uns hier als Produzent und Opfer seiner Vorstellungen entgegen, und es ist eine seiner

16. Vgl. Brecht, „Salomon-Song“. in ders., Gedichte 4, GBA 14, S. 451 f. - Quelle ist François Villons „Double ballade sur le mesme propos“ im Großen Testament (um 1457/58; dt.: „Doppelballade über denselben Gegenstand“). - Brecht spielt in den Psalmen häufiger auf Salomo an und verwendet dessen Kreidekreis-Urteil im Augsburger Kreidekreis (vgl. GBA 18) und im Kaukasischen Kreidekreis (vgl. GBA 8). - Caesars Ausspruch „auch du, mein Sohn!“ ist in Suetons KaiserBiographien De vita Caesarum (dt.: Die zwölf Cäsaren, zweite, neu durchgesehene Auflage nach der Übersetzung von Adolf Stahr, Berlin, Propyläen 1922) in Kapitel 82 der Cäsar-Biographie überliefert. Zum Tod des Sokrates vgl. Brechts Erzählung „Der verwundete Sokrates“, in ders. (Bd 18).

17. Vgl. Brecht, „Die Ballade von den Prominenten“. in ders., Gedichte 1, Sammlungen, 1918-1938 (= Bd. 11 der Großen kommentierten Berliner und Frankfurter Ausgabe; im folgenden kurz GBA 11), hrsg. von Werner Hecht, Jan Knopf, Werner Mittenzwei und Klaus-Detlef Müller, Berlin/ Weimar/ Frankfurt a. M., Aufbau und Suhrkamp, 1993, S. 146 f. Kurt Weill vertont den Song erstmals 1928 für die Dreigroschenoper.

18. Vgl. Brecht, Kommentar zu „Die Songs der Dreigroschenoper“, in ders., Gedichte 1, GBA 11, S. 331-347. Der „Salomon Song“ entstand bereits 1928 für die Dreigroschenoper mit dem Titel „Salomo-Song“ und ist in Die Songs der Dreigroschenoper (1928) mit vier Strophen unter dem Titel Die Ballade von den Prominenten enthalten. Eine Neufassung erfolgte 1937 für den Druck der Dreigroschenoper in Gesammelte Werke mit dem Titel „Salomon-Song“. 
„schwierigsten Aufgaben, aus der Welt des Gedankens in die wirkliche Welt herabzusteigen“. ${ }^{19}$ Mythische Figuren werden hier wie Gestalten der historischen Ereignisgeschichte behandelt. Die Strophe über den heiligen Martin findet erst in den Drucken von Mutter Courage und ihre Kinder (1939) wieder Berücksichtigung (als Strophe 4):

\footnotetext{
Der heilige Martin, wie ihr wißt

Ertrug nicht fremde Not.

Er sah im Schnee ein' armen Mann

Und er bot seinen halben Mantel ihm an

Da frorn sie alle beid zu Tod.

Der Mann sah nicht auf irdischen Lohn!

Und seht, da war es noch nicht Nacht

Da sah die Welt die Folgen schon:

Selbstlosigkeit hat ihn so weit gebracht!

Beneidenswert, wer frei davon! ${ }^{20}$
}

Brechts Verfahren, einen Mythos als ,moralische Erzählung“ zu lesen, wirkt im Ergebnis zynisch: „Ein halber Mantel, lieber Martin, nützt niemandem etwas.“ Mit Blick auf Brechts Prosa hat die Forschung entsprechend einen „Proze $\beta$ der Entmythisierung“ konstatiert. ${ }^{21}$ Ein solcher Stimulus lässt sich analog auch anhand der Lyrik beobachten, wobei Brechts Varianten bei den prominenten, im allgemeinen kulturellen Wissen verankerten Mythen besonders ins Auge stechen. ${ }^{22}$ Dabei muss uns nicht verwundern, dass seine Revisionen bisweilen einen plakativ-raubatzigen Ton anschlagen - die Realität, die ihm entgegenschlug, war auch so.

Christliche Mythen treten in Zyklen (wie den „Visionen“ der „Steffinschen Sammlung“) sowie in Einzelgedichten auf, und wenn es nicht Mythen sind, die alludiert werden, so sind es doch „Mytheme“, die aufscheinen, Bausteine beziehungsweise Spurenelemente von Mythen (analog zu den Weissagungen im Christentum), die sich biblisch referenzialisieren lassen: „Brechts Bilder sind teilweise dem christlichen Mythos verpflichtet“, teilweise werden sie aber auch ins „Aktuell-Politische“ transformiert. ${ }^{23}$ So wird der Auferstehungsglaube (vgl.

19. Vgl. Karl Marx, Friedrich Engels, Die deutsche Ideologie. In dies., Werke. Institut für MarxismusLeninismus beim ZK der SED. Bd. 3. Berlin, Dietz, 1978, S. 432.

20. In einer Vierstrophenfassung (ohne Martin-Strophe) ging der Text 1939 in das Stück Mutter Courage und ihre Kinder ein und wurde dann, allerdings mit Varianten, als „Salomon Song“ 1948 publiziert.

21. Vgl. Franco Buono, „Odysseus...“, S. 61-91.

22. Martin Vöhler argumentiert in einem Aufsatz über Mythenkorrekturen bei Pindar, dass Pindar einen größeren Gestaltungsspielraum genutzt habe als Brecht: „Im Verhältnis zu Brechts Berichtigungen alter Mythen erscheint Pindars Mythenerzählung [scil. des PelopsMythos] außerordentlich voraussetzungsreich und komplex.“ Vgl. Martin Vöhler, „Ich aber: Mythenkorrekturen in Pindars 1. Olympie“, in Martin Vöhler, Bernd Seidensticker (Hrsg.), Mythenkorrekturen. Zu einer paradoxalen Form der Mythenrezeption, Berlin/ New York/ de Gruyter, 2005, S. 19-35. Hier: S. 31.

23. Brecht, Kommentar zu „Visionen“, in ders., Gedichte 2, Sammlungen, 1938-1956 (= Bd. 12 der Großen kommentierten Berliner und Frankfurter Ausgabe; im folgenden kurz GBA 12), hrsg. von Werner Hecht, Jan Knopf, Werner Mittenzwei und Klaus-Detlef Müller, Berlin/ Weimar/ Frankfurt a. M., Aufbau und Suhrkamp, 1993, S. 397. 
Matthäus 27, 52 f.) nicht mit der Resurrectio der Heiligen, sondern mit dem Verwesungsgeruch des Krieges konnotiert: „Allenthalben sah man geöffnete Gräber“, heißt es zu Beginn der „Visionen“, und auch die Hure Babylon kommt mit „Gestank“ nieder und gebiert den Krieg. ${ }^{24}$

Die im Alten Testament erwähnten Städte Sodom und Gomorra, die synekdochisch für den Sittenverfall stehen können, werden bei Brecht auf „unsere Städte“ Berlin und London gemünzt, die durch vergleichbare „Sünden“ ganz „unbewohnbar“ geworden und zugleich doch „unverlaßbar“ geblieben seien. ${ }^{25}$ „Die Hölle“, heißt es in einem späteren Gedicht, „ist eine Stadt, sehr ähnlich London“. ${ }^{26}$ Brechts Reinterpretation biblischer Mytheme führt hier zu einer radikalen Deutung, da er alle Städter zu Höllenbewohnern erklärt, ob sie für das moralisch Gute optiert haben oder nicht. Sogar diejenigen, die gegen das Leid der anderen Vorsorge zu treffen suchen, finden das eigene Glück nicht, sondern landen in der Verdammnis: Die von Franco Buono apostrophierte „materialistische Psychologie“ zeitigt hier Implikationen von ernüchternder Konsequenz. Eine Theodizeefrage stellt sich vor diesem Bezugsrahmen nicht oder anders oder wird apriori negativ beantwortet: Wir leben in der schlechtesten aller Welten, und daran ändern auch die Heilsbotschaften der kommunistischen ,Klassiker nichts.

Seit Beginn seiner Exilzeit plante Brecht, tradierte Mythen einer Revision zu unterziehen. ${ }^{27}$ Nur Weniges wurde ausgeführt, aber eine „Auflistung“ im Nachlass lässt erkennen, dass Brecht den Fundus der christlichen und der heidnischen Mythen (der griechisch-römischen Antike) großzügig erweiterte: um Wilhelm Tell und Wallenstein, um die Brüder Karamasow und Macbeth, um Kolumbus und Kleopatra. ${ }^{28}$ Die Idee, Mythen zu ,verbessern', hatte zuvor schon Franz Kafka realisiert und damit den Gleichnischarakter des Mythos ins Absurde verschoben. Brecht demonstriert gegen Kafka, dass Mythen weder irrational noch paradox sein müssen, weder vormodern noch antimodern. Mit der Anmutung archaisierender Sprechweisen und profaner Topik verknüpft sich bei ihm auch kein existenzial-ontologisches Wahrsprechen transhistorischer Wirkungsmächte, zu dessen (und deren) geheimen Quellen ein visionäres Ich Zugang hätte, aus denen es Kraft schöpfte - sondern es ist ein Sprechen sehenden Auges, mit klarem Blick, aus der Ohnmacht der historischen Situation heraus, die jedermann erfahrbar ist. Ein Mythem der Aufklärung wird hier beschworen, ein Glaubenssatz, als dessen Aufkündigung das erzwungene Exil erlebt wird. Brecht selbst hat das Verfahren, die Geschichtsschreibung oder Überlieferung kritisch zu

24. Vgl. Brecht, „Aus den Visionen“, in ders., Gedichte 2, GBA 12, S. $104 \mathrm{f}$.

25. Vgl. Brecht, „Untergang der Städte Sodom und Gomorra“, in ders., Gedichte 4, GBA 14, S. 247.

26. Vgl. Brecht, „Hölle“, in ders., Gedichte 4, GBA 14, S. 404 f. Hier: S. 404.

27. Vgl. dazu Alexander Honold, „Odysseus in korrigierter Haltung. Entstellungen des Mythos bei Kafka, Brecht, Benjamin und Adorno/ Horkheimer“. in Mythenkorrekturen, S. 317-329.

28. Vgl. Brecht, Kommentar „Zweifel am Mythos“, in ders., Prosa 4, Geschichten, Filmgeschichten, Drehbücher 1913-1939 (= Bd. 19 der Großen kommentierten Berliner und Frankfurter Ausgabe; im folgenden kurz GBA 19), hrsg. von Werner Hecht, Jan Knopf, Werner Mittenzwei und KlausDetlef Müller, Berlin/ Weimar/ Frankfurt a. M., Aufbau und Suhrkamp, 1993, S. 663. 
befragen und neu zu deuten, erstmals in „Tod des Cesare Malatesta“ verwandt. ${ }^{29}$ Mit Beginn des Exils 1933 wird es zu einem wichtigen Darstellungsmuster, um den „Zweifel am Mythos“ zu artikulieren. ${ }^{30}$

\section{Brechts mythisches Ich und seine Lyrik der Verstandesbildung}

Der vornehmste Gegenstand der Mythisierung war für einen selbstbewussten Dichter wie Brecht von jeher sein lyrisches Ich. Brecht hat früh schon begonnen, die eigene Person zu mythisieren und zu mystifizieren: „Ich bin aus den schwarzen Wäldern“, dichtete das Augsburger Stadtkind im Stile François Villons, so dass man auch von einem „Mythos Brecht“ gesprochen hat. ${ }^{31}$

Dieser mythische Brecht ist erfındungsreich: „Ich bin ein Stückeschreiber“, heißt es da etwa 1935, „Ich bin der Sattler“ (aus der Kriegsfibel), „Ich bin der Glücksgott“ (1941), oder einfach: „Ich erklettere das Gebirge“ (1934). Der akademische Vorbehalt, dass man ein lyrisches Ich nicht auf die Person des Dichters beziehen dürfe (und oftmals auch gar nicht könne), wird zurecht erhoben (und hier auch respektiert). Es ist jedoch nicht zu leugnen, dass Lyrik für Brecht ein Medium der Selbstbegegnung und Selbststilisierung darstellte und dass wir aus den Gedichten der Exilzeit viel über die Lebenswirklichkeit des Dichters erfahren (zu können meinen...), wenngleich in gefilterter Form. Wir kommen daher nicht umhin, sie (auch) als Quelle zu lesen, allem Vorbehalt zum Trotz.

Viele Schriftstellerkollegen Brechts sind am Exil zerbrochen oder haben schon die Flucht nicht überlebt. Wir kennen sein Gedicht auf den tragischen Tod Walter Benjamins: „Zum Freitod des Flüchtlings W. B.“ (1941). ${ }^{32}$ Eben noch hatten die Freunde über Kafka gestritten, jetzt ist Benjamin aus dem Leben geschieden, tot von eigener Hand. Ein anderes Gedicht ist Margarete Steffin gewidmet, „gestorben an der Erschöpfung auf der Flucht vor Hitler“. ${ }^{33}$ Brecht überlebte - vielleicht gerade dank seiner intellektuellen Mobilität und seiner Neigung zur Selbststilisierung. Das Bild, das er zwischen 1933 und 1945 von sich entstehen ließ, ist durchaus stimmig, mag es auch vielgestaltig sein. Vor allem aber ist es abwechslungsreich, von großer Vitalität und Produktivität getragen.

29. Vgl. Brecht, „Tod des Cesare Malatesta“, in ders., Prosa 4, GBA 19, S. 183-188.

30. Vgl. ebd., den Brecht-Kommentar zu „Zweifel am Mythos“, in ders., Prosa 4, GBA 19, S. 662-665. - Vgl. u.a. die „Fragen eines lesenden Arbeiters“ (in Brecht, Gedichte 2, GBA 12, S. 29 f.), das Romanfragment Die Geschäfte des Herrn Julius Caesar (in Brecht, Prosa 2, GBA 17, S. 163-198) sowie die „Kalendergeschichten“ „Der Mantel des Ketzers“ und „Der verwundete Sokrates“ (in Brecht, Prosa 3, GBA 18, S. 374-382 und 410-425).

31. Neben Otto Mann (vgl. Fußnote 4) waren dies etwa Franco Buono (vgl. ders., Brecht, 1917-1922, Jugend, Mythos, Poesie, Deutsch von Bernt Ahrenholz und Ursula Ladenburger, Göttingen, Steidl 1988) und Frank D. Wagner (vgl. ders., Mythos der Nation, Bronnen und Brecht, Würzburg, Königshausen und Neumann 2015). - Vgl. auch das Gedicht „Ich, Bertolt Brecht, bin aus den schwarzen Wäldern“ („Vom armen B. B.“) in ders., Gedichte 1, GBA 11, S. 119 f. Das Gedicht stammt aus dem Anhang der Hauspostille. Vgl. dazu den Brecht-Kommentar, ebd., S. 324.

32. Vgl. Brecht, „Zum Freitod des Flüchtlings W. B.“, in ders., Gedichte 5, GBA 15, S. 48.

33. Vgl. Brecht, „Kinderkreuzzug 1939“, in ders., Gedichte 5, GBA 15, S. 343. 
Dieser Mythos Brecht ist ein Kosmos für sich und kann hier nur ansatzweise erschlossen werden. Daher sei hier knapp und thesenhaft eine Topologie der Brecht'schen Mytheme erstellt, wobei gelten soll, dass die Stationen des ebenfalls vielgestaltigen - Exils mit Brechts Selbstbild eng korreliert sind:

1. Der Flüchtling Brecht stilisiert sich als Antipode. Dabei wird sein Gegenspieler Hitler mit einer eigenen Topik belegt (der „Anstreicher“), die im Widerstand gängige Münze war. Der Flüchtling selbst dichtet im Bewusstsein seiner Unterlegenheit („In Erwägung unsrer Schwäche“), aber auch seiner Erfahrung: der „Weg des Laotse in die Emigration“ zeitigt eben auch Wissensbestände, die er anderen voraus hat und in Stärke verwandeln kann. ${ }^{34}$

2. Der Flüchtling Brecht stilisiert sich als Seher. ${ }^{35}$ Unter dem Eindruck seines marxistischen Lehrers Karl Korsch und seines Freundes Walter Benjamin wendet sich Brecht gegen eine „falsche Augenscheinlichkeit des Mythos“, indem er den „Vorzeitlichen Mythos“ als „Stoff des Fortschritts“ reinterpretiert. ${ }^{36}$ Brecht erscheint hier als Dichterseher der Zeitläufte, der mitten im Weltgeschehen steht beziehungsweise die politischen Entwicklungen aus der besseren Perspektive der erzwungenen Peripherie beobachtet:



Das wörtliche und das metaphorische Sehen ergänzen sich hier zur ,inneren Schau der (kommunistischen) ,Klassiker‘, deren Visionen politische Ereignisgeschichte geworden sind. Das Gedicht vom Bau der Moskauer Metro ist das genaue Gegenbild des - weitaus berühmteren - Gedichts „Fragen eines lesenden Arbeiters“ (ebenfalls 1935 verfasst). Während letzteres aber bemängelt, dass die Geschichtsschreibung von den wahren „Bauherren“ der großen Kulturgüter und mythischen Stätten keine Kunde geben wolle (denn solche „historischen Leistungen“ würden ausschließlich den „großen Individuen“ zugeschrieben), rückt ersteres die Arbeiter als die tatsächlichen „Bauleute“ ins Zentrum des

34. Vgl. Brecht, „Legende von der Entstehung des Buches Taoteking auf dem Weg des Laotse in die Emigration“, in ders., Gedichte 2, GBA 12, S. 32-34.

35. Vgl. Franco Buono, „Bemerkungen...“, S. 105. Da sich Buono zurecht von einer Reduzierung Brechts auf „einen moralistischen Seher“ (ebd.) distanziert, könnte man auch von ıBrecht als Zeitdiagnostiker' sprechen. Während aber die seit Augustinus' De civitate Dei (XVI, 9) reflektierte Denkfigur des Antipoden mythische Qualität annehmen kann, muss der Zeitdiagnostiker erst noch zum Mythem im eigentlichen Wortsinn auserzählt werden.

36. Ich folge hier der Argumentation von Franco Buono, „Odysseus...“, S. 67 u. 71 . Buono wiederum bezieht sich auf Theodor W. Adorno und Max Horkheimer, Dialektik der Aufklärung. Philosophische Fragmente, Amsterdam, Querido, 1947, S. 46.

37. Brecht, „Inbesitznahme der grossen Metro durch die Moskauer Arbeiterschaft am 27. April 1935“, in Gedichte 2, GBA 12, S. 43-45. Hier: S. 45. 
Geschehens: Nicht nur die Vollendung der Metro wird gefeiert, sondern auch deren „Inbesitznahme“ durch die neue Klasse der Werktätigen, die sich hier mit dem von ihr aufgeführten Bau selbst feiert. ${ }^{38}$

In gut propagandistischer Manier beschreibt Brechts Dichterseher diese „Inbesitznahme“, die er selbst nur vom Hörensagen kannte: „Wir hörten“, heißt es denn auch gleich zu Beginn der ersten Strophe. ${ }^{39}$ Brecht war nicht vor Ort, und dennoch kann man seinem Gedicht mangelnde Distanz attestieren - denn es verklärt den Vorgang zum Heilsgeschehen.

Brechts Verfahren, die Mythen und Masken des Selbst im Kontext seiner Lyrik zu erörtern, war erfolgreich. Der Dichter adressierte dabei nicht nur den ,gebildeten 'Leser: Sein poetisches Gesprächsangebot funktionierte weithin, denn seine Lyrik wurde von seinen Erstrezipienten sowohl als innovativ wie auch als anschlussfähig empfunden. Dabei muss man, um ihren literarischen Charakter zu verstehen, ihre Sprechsituation und ihre Voraussetzungssituation genauer in den Blick nehmen:

Nun, Timon, Menschenfeind im Hades, sag:

Nacht oder Tag, was bringt dich mehr zum Toben?

„Noch mehr haß ich das Dunkel als den Tag

Sind doch hier unten mehr von euch als droben." ${ }^{40}$

Diese vier Zeilen des titellosen Dialoggedichts aus dem Jahre 1940 bilden schon den ganzen Text und wirken nachgerade voraussetzungslos. Der Kunstgriff des (scheinbar) spontanen Sprechens und der umstandslosen Wechselrede (bei der nur ein Sprecher markiert sein muss) ist uns dabei wohlvertraut. Er begegnet uns beispielsweise in der Sturm-und-Drang-Phase, etwa in Goethes „Prometheus“-Gedicht („Bedecke deinen Himmel, Zeus“), wo der Redegegenstand einerseits mythisch entrückt ist, durch die Sprechsituation wiederum aber vergegenwärtigt wird, und zwar so, dass Sprechsituation und besprochene Situation zusammenzufallen scheinen. Bei der besprochenen Situation (als der dargestellten Welt) des Brecht'schen Vierzeilers fällt auf, dass das traditionelle Hades-Mythem variiert wird: Der Tag als Synonym des Lebens brachte dem Menschenfeind immerhin noch Abgänge, über die er sich freuen konnte. Die Nacht als Synonym des Todes hingegen bringt ihm nur noch Zugänge. Deshalb muss Timon den Hades im doppelten Sinne als Verbannungsort empfinden: Sein Aufenthalt dort gleicht einer eigens auf ihn gemünzten Höllenqual. Hätte er eine Stätte der Seligen aufsuchen dürfen, so wäre er vielleicht besser zurechtgekommen - denn von den Seligen gibt es nur wenige.

38. Vgl. dazu Siegfried Mews, „Fragen eines lesenden Arbeiters“, in Jan Knopf (Hrsg.), Brecht-Handbuch, Band 2, Gedichte, Stuttgart/ Weimar, Metzler, 2001, S. 281-284, hier: S. 282. - Auf einem anderen Blatt steht, dass die Arbeiter selbst und ihre Verhältnisse in der Sowjetunion verklärt werden, wie etwa auch das Gedicht „Der große Oktober“ demonstriert (im Untertitel „Zum zwanzigsten Jahrestag der Oktoberrevolution“). Vgl. Brecht, in ders., Gedichte 2, GBA 12, S. 45 f.

39. Brecht, „Inbesitznahme...“, S. 43.

40. Vgl. Brecht, „Nun, Timon, Menschenfeind im Hades, sag“, in ders., Gedichte 5, GBA 15, S. 14. 
Auch bei dem folgenden Poem fällt die Sprechsituation mit der besprochenen Situation zusammen - entsprechend sind nur Tempora der besprochenen Welt (Präsens, Perfekt - mit dem Leittempus Präsens) beobachtbar. ${ }^{41}$ Sie sind in dieser Konstellation typisch für die Erörterung ebenso wie für die im Gestus der Apostrophe angelegte Wechselrede:

\footnotetext{
DER KRUMME BOGEN und der Köcher hier

Der so viel Todespfeile ausgesendet

Sie sind hier aufgehängt, o Phoibos, dir

Als Weihgeschenk, von Promachos gespendet.

Die Pfeile aber kann er dir nicht weihn

Da sie, des Schlachtgewühles blutige Schrecken

Ja in den Herzen vieler Männer stecken

Ein Gastgeschenk und eine Todespein. ${ }^{42}$
}

Während in den „Sokratikoi Logoi“ hauptsächlich Sokrates agiert und spricht sowie den Mythos ins Spiel bringt, haben die Brecht'schen Krypto-Dialoge häufıg keinen erkennbaren lyrischen Sprecher. Das verleiht den Versen eine gewisse Universalität, und fast könnte man sie auf beliebige Situationen, Gesellschaftslagen, Topoi applizieren - wäre da nicht Phoibos, ihr Adressat. Dennoch: Ein Leser des Jahres 1940 kann das Gedicht vom „Krummen Bogen“ ohne weiteres auf die eigene Kriegssituation beziehen. Daran ändert auch die Tatsache nichts, dass im Zweiten Weltkrieg niemand mehr mit Pfeil und Bogen kämpfte oder sich etwas von Promachos versprechen durfte. Denn wir erwarten solche dichterische Sprache geradezu, wir sind es nachgerade gewohnt, uns mit mythischen Gestalten und ,verbrauchten“ Bildfeldern zu arrangieren. „Neue Bildfelder hingegen werden äußerst selten gestiftet, zumal ,kühne Metaphern, die einer modernen Wirklichkeit vielleicht besser entsprächen, nicht selten als störend empfunden werden. Lieber belässt man es bei archetypischen Imagines, die sich der Leser mühelos ins Heute übersetzt. ${ }^{43}$ Analog zum Epischen Theater als einer Form der Vernunftkritik, wollte auch Brechts Lyrik der Verstandesbildung dienen und aufklärerisch wirken. Ein drittes Beispiel mag uns dies belegen:

DU SPEER AUS ESCHENHOLZ, hier bleibe stehn

Du Männermörder, denn nie will ich wieder

Von deiner erzenen Spitze nieder

Das Blut der Feinde gräßlich tropfen sehn.

Hier in Athenens glanzerfülltem Haus

Dem hochgetürmten Tempel bleib und liege

Und ruf die Mannestugend und die Siege

Des Echokratidon aus Kreta aus. ${ }^{44}$

In diesem Gedicht wird ein Attribut der Pallas Athene angerufen; die Lanze zu schwingen (griech. „pallein“), gehört dabei zu den Kernkompetenzen der „Göttin

41. Die Terminologie nach Harald Weinrich, Tempus. Besprochene und erzählte Welt, 4. Aufl. Stuttgart, Berlin, Köln/ Mainz, Kohlhammer, 1985 (Sprache und Literatur, 16).

42. Vgl. Brecht, „Der krumme Bogen“, in ders., Gedichte 5, GBA 15, S. 14.

43. Vgl. dazu Harald Weinrich, Sprache in Texten, Stuttgart, Klett, 1976.

44. Vgl. Brecht, „Du Speer aus Eschenholz“, in ders., Gedichte 5, GBA 15, S. 14. 
der klugen Kriegsführung“. ${ }^{45}$ Im Gegensatz zu Ares jedoch, welcher Krieg um jeden Preis führt, steht Athena „für den mit Beherrschung, Verstand und Strategie geführten Kampf“. ${ }^{46}$ Im Parthenon nun, ihrem wichtigsten Tempel, soll der „Speer aus Eschenholz" künftig verbleiben; und die Männer sollen ihre Kräfte fortan in sportlichen Wettkämpfen messen. Dies entspricht dem Wesen der Pallas Athene als Friedensgöttin und Beschützerin der Polis als dem Mittelpunkt einer (neuen) gesetzlichen Ordnung, mittels deren die freien Bürger selbst über ihre Geschicke bestimmen und sich nicht mehr von Schicksalsmächten dominieren lassen.

Doch wer spricht hier eigentlich? Zum einen könnte Athena selbst der Sprecher sein, dann nämlich, wenn sie - etwas gravitätisch - von sich in der dritten Person spräche. Denn wer anderes hätte Macht über ihre Lanze, wenn nicht sie selbst? Nur sie kann doch verfügen, dass eine Kriegswaffe künftig in ihrem Tempel auf der Akropolis verbleiben soll. Nur sie hat die Macht, dem Kriegsgott Ares sowie den anderen Göttern Paroli zu bieten. Und nur sie setzt sich erfolgreich gegen das System der Blutrache durch, indem sie sich bei den Erinyen, den späteren Eumeniden, Unterstützung für die von ihr neu geschaffene, unabhängige Gerichtsbarkeit holt. ${ }^{47}$

In den folgenden Versen ist die Sprechsituation eindeutiger:

Einst war ich das geschwungene Hörnerpaar

Von einer wilden Ziege, wie sie klettern

Auf hohem Fels und im gekrausten Haar

War ich gar oft bekränzt mit grünen Blättern.

Nun hat ein Drechslermeister mich zum Bogen

Für Nikomachos schön zusamm'geschweißt

Hat mich geschickt geglättet und bezogen

Mit einer Rindersehne, die nicht reißt. ${ }^{48}$

Hier wird nicht auf ein bekanntes Mythem referiert, sondern es wird im Gestus der Mythen der Alten erzählt, als ob damit ein altehrwürdiger Mythos transformiert werden solle. Das geschwungene Hörnerpaar einer Ziege wird zu einem Bogen, einer Jagdwaffe, umgebaut, und stolz berichtet es/er von dieser Metamorphose, die der Bogen einem „Drechslermeister“ verdankt. Ob sich damit jetzt vielleicht Jagd auf Ziegen machen lässt? Der Hinweis auf Nikomachos, über den wir nichts Bestimmtes wissen, lässt uns an Nikomachos von Gerasa denken, oder auch an den Sohn des Aristoteles („Nikomachische Ethik“), den literarischen Nachlassverwalter seines berühmten Vaters. ${ }^{49}$ Geht es um ethische Fragen, so

45. Eric M. Moormann, Wilfried Uitterhoeve, Lexikon der antiken Gestalten. Mit ihrem Fortleben in Kunst, Dichtung und Musik, übersetzt von Marinus Pütz, Stuttgart, Kröner, 1995 (Kröners Taschenausgabe, 468), S. 137.

46. Ebd.

47. Vgl. dazu Christian Meier, Die Entstehung des Politischen bei den Griechen, Frankfurt a. M., Suhrkamp, 1980, S. 144-246. - Hinter Echekratidos aus Kreta vermutet der Brecht-Kommentar Echekradites aus Pharsalos, einen Olympiasieger im Reiten. GBA 15, S. 325.

48. Vgl. Brecht, „Einst war ich das geschwungne Hörnerpaar“, in ders., Gedichte 5, GBA 15, S. 15.

49. Der Kommentar vermutet hingegen, es könne sich um den Maler Nikomachos von Thelen handeln. Vgl. GBA 15, S. 325. 
irritiert hier, dass das lyrische Ich mit Stolz von seiner Verwandlung zu sprechen scheint - als wolle es sich seiner neuen Funktion und Aufgabe freuen. Der Umbau eines Hörnerpaars von einem lorbeerbekränzten Körperschmuck zu einer Jagdwaffe kommt einer Veredelung gleich, denn statt der Ziege dient man jetzt dem Menschen. Der unbekümmerte Tonfall des Sprecher-Ichs zeitigt im Ergebnis vielleicht das eigentliche Skandalon - und ist zugleich womöglich als Wink auf deutsche Betriebe lesbar, die sich plötzlich als „kriegswichtig“ wahrnahmen.

Es gibt viele Möglichkeiten, den Mythos zu befragen, beispielsweise hinsichtlich seiner Historizität, seiner Sakralität, seiner Realität. So tut es der „lesende Arbeiter“ in Brechts politisch-sozialem Zeitgedicht „Fragen eines lesenden Arbeiters“ (1935). Anscheinend fragte Brecht durchaus nach der historischen Faktizität ,hinter dem Mythos. Jedenfalls haben ihn viele seiner Leser so verstanden, als sie seine Gedichte der Exilzeit interpretierten und zu diesem Behufe die historische Faktenlage rekonstruierten. So hat es beispielsweise Edgar Neis getan, als er Brechts Gedicht „Fragen eines lesenden Arbeiters“ mit dem kulturellen Wissen unterfütterte, auf das Brecht hier augenscheinlich zu sprechen gekommen war. Hier zunächst einige Verse aus Brechts Gedicht:

Fragen eines lesenden Arbeiters

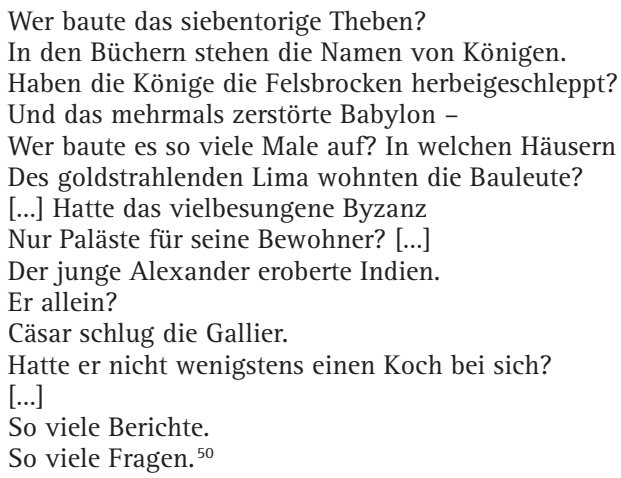

Der Aufsatz des Interpreten Neis trägt nun wie ein Stellenkommentar heran, worauf Brecht angespielt habe, zum Beispiel auf den Phoinikier Kadmos und auf Nebukadnezar II.:

Der Phoinikier Kadmos erbaute 1519 v. Chr. das siebentorige Theben; [...] Nebukadnezar II., der von 605-562 v. Chr. regierte, war der mächtigste König des neubabylonischen Reiches; er baute das mehrmals zerstörte Babylon wieder auf und aus; 587 eroberte er Jerusalem. ${ }^{51}$

So habe es Brecht „in den Geschichtsbüchern“ gelesen, und „so steh[e] es heute noch darin“. Und so habe Brecht es folglich in sein Gedicht getragen: „Daher seine

50. Edgar Neis, „Fragen eines lesenden Arbeiters (Brecht)“, in Politisch-soziale Zeitgeschichte, Bange, Hollfeld, S. 61-66. Hier: S. 61.

51. Ebd., S. $61 \mathrm{f}$. 
Feststellungen und Fragen." ${ }^{2}$ Aber die auf Mythologie begründete Dichtung Brechts fragt nach Zusammenhängen ,hinter den historischen Begebenheiten und Ereignissen, die einer ,Ordnung der Dinge ' hinter den ,Verhältnissen“ folgen. Während sich die Mythologie seit alters und ihrem Ursprung nach in einer Zeit verliert, in die keine historische Kunde zurückreicht, thematisiert Brechts Lyrik Konstellationen und Ereignisse, die der genauen Datierung noch erreichbar sind. Ob er aber aus dem Historischen seine Folgerungen zieht, oder aus dem Vorhistorisch-Mythologischen seine Dialektik ableitet, steht für uns außer Frage. Denn offenbar verfälscht die verbürgte Erzählung die im Mythos angelegte Losung, die Brechts Lyrik wieder hervorzaubert, indem sie sich auf die Verhältnisse bezieht, unter denen die Mythen entstanden sind. Jede Mythos-Rezeption steht - zumal "für einen Autor mit so fundamental funktional ausgerichteter Poetik“ 53 - unter dem Zeichen der Verwendbarkeit und Übertragbarkeit der mythischen Erzählung. Wozu lässt sie sich einsetzen, welche Erkenntnis lässt sich aus ihr gewinnen, was sagt sie uns über die ,Natur der Verhältnisse? Erzählt sie uns, ins Heute getragen, alte Wahrheiten - oder neue Dummheiten? Kommen von den „neuen Antennen“ die „alten Dummheiten“ oder wird die ewige „Weisheit“ verwandelt „von Mund zu Mund weitergegeben“? ${ }^{54}$

Als anthropomorphe Weltbilder haben Mythen und Mytheme immer auch die Lebenspraxis der Menschen beeinflusst und mitbestimmt; als Medium der Sinnstiftung waren sie einer Form der Daseinsvorsorge äquivalent, auf die man sich auch unter widrigsten Bedingungen einstellen konnte. Ob Mythen solche Kräfte heutzutage noch entfesseln könnten? Vielleicht, denn ein Mythos benötigt nur den Zauber der Verwandlung, um uns zu erreichen und uns von unserer Erdenschwere zu erlösen - in welcher Gegenwart auch immer wir leben. Ein mythisches Narrativ bildet das allseits anschlussfähige Tertium comparationis von Erfahrungswirklichkeit und wissenschaftlicher Expertise, von Geschichte und Offenbarung, von Immanenz und Transzendenz; es könnte sich auch künftig bewähren, ebenso, wie sich Religion und Astrologie, Wunderglaube und Heilsversprechen in der Welt der Ratio behaupten. Wir müssen daher mit der Möglichkeit rechnen, dass unter den Bedingungen der Moderne die Chancen für den Mythos wieder steigen: Denn wir irren vorwärts.

52. Ebd., S. 62.

53. Vgl. Christel Hartinger, „Lehrgedicht“, in Jan Knopf (Hrsg.), Brecht-Handbuch, Band 2, Gedichte, Stuttgart/ Weimar, Metzler, 2001, S. 397-404, hier: S. 400.

54. Vgl. Brecht, „Die neuen Zeitalter“, in Gedichte 5, GBA 15, S. 102. 